\title{
SOBRE EL CARÁCTER ALAS EN BELGICA ANTARCTICA JACOBS (DIPTERA: CHIRONOMIDAE) REGISTRADO ERRÓNEAMENTE EN LA LITERATURA ${ }^{1}$
}

\author{
ON THE CHARACTER WINGS IN BELGICA ANTARCTICA JACOBS (DIPTERA: \\ CHIRONOMIDAE) ERRONEOUSLY REGISTERED IN THE LITERATURE
}

Vicente Pérez ${ }^{2}$

Belgica antarctica Jacobs, 1900, es un mosquito de tamaño pequeño (macho: 1,6-2,5 mm de longitud y 0,3-0,5 mm de ancho; hembra: 1,5-3,2 $\mathrm{mm}$ de longitud y 0,3-0,85 mm de ancho) (Wirth \& Gressitt 1967). Fue descrito brevemente por Jacobs (1900), sobre la base de una captura de 50 adultos (Usher \& Edwards 1984) procedente del estrecho de Gerlache, Antártida chilena. Stuardo (1946), la señala como una de "las especies más australes de nuestra fauna dipterológica". A juicio de Severin (1906), el descubrimiento de esta especie representó el mayor acontecimiento de la Expedición Antártica Belga (1897-1899).

En los insectos, el carácter "alas" tiene una importancia biológica y sistemática considerable (Séguy 1959), toda vez que son éstos los únicos animales que poseen órganos propios para el vuelo. Su número, estructura, forma y venación son caracteres que se consideraron históricamente al acuñarse los nombres con el elemento compositivo -pteron en no menos de 30 de 39 órdenes (Gillott 2005).
El modelo díptero, con un par de alas mesotorácicas para el vuelo y un par de balancines metatorácicos para el equilibrio, ostenta la capacidad de vuelo más perfecta dentro de los insectos alados.

En Belgica antarctica ambos sexos son alados, aunque las alas están reducidas a cortos y angostos sacos, y carecen de balancines. Ambas características están claramente expuestas en las descripciones históricas (Jacobs 1900, 1906a, 1906b; Rübsaamen 1906) y en las ilustraciones (Rübsaamen 1906; Wirth \& Gressitt 1967; Hashimoto 1976).

Los quironómidos constituyen una gran familia, de amplia distribución, de más de 5.000 especies de pequeño tamaño, cuyos adultos con habitus de mosquito, aunque no se alimentan; forman a menudo enjambres masivos en la vecindad del agua. Los hábitos de vuelo en enjambre de los quironómidos, así como su escaso peso, podrían rendirle ventajas ecológicas y adaptativas, particularmente a su dispersión por medio del viento (Edwards 1931).

La mayoría de las especies de quironómidos son terrestres, con larvas acuáticas o terrestres.

1 Perteneciente al Proyecto Interno "Biodiversidad de la Entomofauna de la Región de Magallanes" (V. Pérez, Investigador Principal).

2 Laboratorio de Entomología, Instituto de la Patagonia, Universidad de Magallanes. Casilla 113-D. Punta Arenas. 
Alrededor de 12 géneros con 50 especies son marinos y están restringidos a las zonas litorales. Jacobs (1900) anota que las larvas de Belgica antarctica se encontraron en pequeñas charcas producidas por el derretimiento de nieve; estas larvas terrestres (por oposición a marinas) son conocidas desde hace mucho tiempo para la parte media de la Península Antártica (lado occidental), pero nuevamente registradas en las Shetlands del Sur (varias islas) (Gressitt 1967); larvas y adultos buscan protección bajo los musgos (Mann 1948).

El objetivo de esta Nota es detectar errores que, sobre el carácter "alas" en Belgica antarctica, registra la literatura especializada, algunos textos clásicos de entomología y documentos oficiales sobre zonas protegidas de la Antártica. La especie se considera importante desde muchos puntos de vista, incluyendo los taxonómicos, ecológicos, biogeográficos y evolutivos, particularmente válidos para el territorio antártico. Para ello, se realizó una exhaustiva revisión de toda la literatura disponible sobre esta especie. En general, fue posible distinguir cuatro errores generales, que pueden ser asociados ya sea con problemas de traducción o a malas interpretaciones de características morfológicas dado el tamaño de los individuos.

\section{ERRORES DETECTADOS Y PROBABLES CAUSAS DE ELLOS}

- Que Belgica Jacobs, 1900, es un género marino: Hashimoto (1976).

Probablemente sea un error de consideración, por cuanto el hábitat se encuentra dentro de lo que muchos autores denominan "Antártica marítima" (por oposición a la zona alejada de la costa marina).

- Que es una mosca (fly) áptera: Lindsey (1940).

Puede ser un error de traducción, ya que Diptera es el orden de los insectos generalmente conocidos como "flies" en inglés. Esta misma situación se presenta en las versiones en castellano de los documentos ASPA en Internet.

- Que es un mosquito (midge) áptero: Españoll Coll (1957, 1968), Imms (1960), Gressitt et al. (1963), Gressitt (1967), Wirth \& Gressitt (1967), Peckhamm (1971), Richard \& Davis (1977), Baust \& Lee Jr. (1987), (ASPA 115 (2000), ASPA 126 (2002).
A esta afirmación pueden haber contribuido el pequeño tamaño de los ejemplares, el desconocimiento de los textos e ilustraciones (dibujos, en vista lateral) de la literatura fundacional de la especie y el oscuro melanismo del tegumento del insecto adulto ilustrado en fotografías modernas en blanco y negro en vista dorsal, tanto en textos como en Internet, que impide observar las pequeñísimas alas de los ejemplares. Este estado del carácter alas en $B$. antarctica, tan reducido, carente de venación, sería un caso de micropterismo dentro del grupo de dípteros de alas reducidas (braquípteros y micrópteros), que no sobrepasa el 0,7\% de las especies actualmente conocidas (Seguy 1959).

En los documentos aprobados internacionalmente como Planes de Manejo para Áreas Especialmente Protegidas en la Antártica [(ASPA 115 (2000), ASPA 126 (2002)], está incluida expresamente Belgica antarctica entre los "valores que deben ser protegidos", pero sin ninguna ilustración de la especie que permita reconocerla.

El mérito de estos documentos radica esencialmente en que valoriza a los artrópodos terrestres para que sean incluidos en los Estudios de Impacto Ambiental, que todavía no han alcanzado un nivel de amplitud y profundidad en Chile.

- Que no presenta dimorfismo sexual: Hashimoto (1976).

Esto sería válido si sólo se refiriera al carácter alas, pero en sentido amplio, en una especie gonocorista, dimorfismo es la existencia de dos formas corporales en una especie (Blakwelder \& Garoian 1986). En B. antarctica, la terminalia del macho se caracteriza por el conspicuo último tergo, casi rectangular, que permite identificar el sexo.

\section{LITERATURA CITADA}

ASPA 115. 2000. Management Plan for Specially Protected Area No. 115. Measure 1. Lagotellerie Island, Margueritte Bay, Graham Island. CEP HANDBOOK 2011 [Committee for Environmental Protection. Secretariat for Environmental Protection] 12 pp. http://ats.aq/ documents/recatt/att201_e.pdf. 10/05/2012

ASPA 126. 2002. Management Plan for Specially Protected Area No. 126. Recommendations VIII-2 (1975); X-6 (1979) (E), XII-5) (1983) (E), 
XIII-7 (11985 (E). Byers Peninsula, Livingston Island, South Shetland Islands. CEP HANDBOOK 2011 [Committee for Environmental Protection. Secretariat for Environmental Protection]. 21 pp. http://www.ats.aq/documents/recatt//att474_e.pdf 10/05/2012

Baust, J. G. \& R.E. Lee Jr. 1987. Multiple tolerance in an Antarctic terrestrial arthropod: Belgica antarctica. Cryobiology, 24(2):140-147

Blackwelder, R.E. \& G.S. Garoian 1986. CRC Handbook of Animal Diversity. CRC Press, Inc. Boca Raton, Florida. 555 pp.

Brues, C.T., A.L. Melander \& F.M. Carpenter 1954. Classification of Insects. Bulletin of the Museum the Museum of Comparative Zoology, 68. 917 pp.

Edwards, F.W. 1931. Chironomidae. Diptera of Patagonia and South Chile. Part II. Fascicle 5:233-324

Españoll Coll, F. 1957. Dípteros. In Martínez-Hidalgo y Terán (Dir.), Enciclopedia General del Mar 2:792-794. Ediciones Garriga S.A. Madrid Barcelona.

Españoll Coll, F. 1968. Dípteros. In Martínez-Hidalgp y Terán (Dir.), Enciclopedia General del Mar 3:815-816. Segunda Edición Ampliada y Puesta al Día. Ediciones Garriga S.A. Madrid - Barcelona.

Gillot, C. 2005. Entomology. Third Edition. Springer. 831 pp.

Gressitt, J.L. 1967. Introduction. In Gressitt J.L. (Ed). Entomology of the Antartica. Antarctic Research Series, 10:1-33. Publisher American Geophysical Union of the National Academy of Sciences-National Research Council. Publication No. 1574.

Gressitt, J.L., R.E. Leech \& K.A.J. Wise 1963. Entomological Investigations in Antarctica. Pacific Insects, 5(1):287-304

Hashimoto, H. 1976. Non biting midges of marine habitats (Diptera: Chironomidae). In L. Chun (Ed.) Marine insects, pp. 377-414. NorthHolland Publiahing Company, Amsterdam.

Imms, A.D. 1960. A General Textbook of Entomology. London: Methuen \& Co. Ltd. [Ninth Edition Reprinted with minor corrections]. $886 \mathrm{pp}$

Jacobs, J.C. 1900. [Diagnoses d'insectes recueillis par l'Expédition Antarctique Belge]. Diptera.
Annales de la Société Entomologique de Belgique, 44:106-107

Jacobs, J.C. 1906a. Diptères. Bibionidae, Chironomidae, Culicidae, Syrphidae, Muscidae, Rhyphidae, Anthomyidae. Expédition Antarctique Belge. Résultats du Voyage du S.Y. Belgica, 1897-1899. Rapports Scientifiques, Zoologie, Annales de la Société Entomologique de Belgique, 10:67-71

Jacobs, J.C. 1906b. [Belgica n. g. et Belgica antarctica n.sp]. In Rübsaamen, E.H. Diptères. Chironomidae. Expédition Antarctique Belge. Resultats du Voyage du S.Y. Belgica, 1897-1898-1899. Rapports scientifiques, Zoologie, Annales de la Société Entomologique de Belgique 44:75-76, pl. IV. www. vliz.be/imisdocs/publications/150491.pdf $10 / 05 / 2012$

Lindsey, A.A. 1940. Biology and biogeography of the Antarctic and subantarctic Pacific. Proceedings of the Sixth Pacific Science Congress, 2:715-721

Mann, G. 1948. Biología de la Antártica Sudamericana.Estudios realizados durante la Expedición Antártica Chilena, 1947. Imprenta Universitaria. Santiago de Chile. 364 pp.

Peckham, V. 1971. Notes on the chironomid midge Belgica antarctica Jacobs at Anvers Island in the maritime Antarctica. Pacific Insects Monograph, 25:145-166

Richards, O.W. \& R.G. Davies 1977. Imm's General Textbook of Entomology. Tenth Edition. Vol. 2. John Wiley \& Sons, New York.

Rübsaamen, E.H. 1906. Diptères. Chironomidae. Expédition Antarctique Belge. Résultats du Voyage du S.Y. Belgica, 1897-1898-1899. Rapports scientifiques, Zoologie, Annales de la Société Entomologique de Belgique 44:75-85, pl. IV et V. w ww.vliz.be/imisdocs/ publications/150493.pdf 24/08/2011

Séguy, E. 1959. Introduction a l'étude morphologique de l'aile des insectes. Mémoires du Muséum National d'Histoire Naturelle. Série A, Zoologie, 21:1-248

Severin, G. 1906. Introduction. Zoologie. In Commision de la Belgica (Dir.), Résultats du voyage du S.Y. Belgica en 1897-1898-1899: sous le commandemment de A. de Gerlache de Gomery. Rapports scientifiques Publics aux 
frais du gouvernement belge, sous la direction de la Commission de la Belgica.

Stuardo, O.C. 1946. Catálogo de los Dípteros de Chile. Ministerio de Agricultura. Imprenta Universitaria. Santiago de Chile. 253 pp.

Usher, M.B. \& M. Edwards 1984. A dipteran from south of the Antarctic Circle: Belgica antarc- tica (Chironomidae) with a description of its larva. Biological Journal of the Linnean Society, 22:19-31

Wirth, W.W. \& J.L. Gressitt 1967. Diptera: Chironomidae (Midges). In Gressitt J.L. (Ed.) Entomology of Antarctica, pp. 197-203. 